\title{
INSURANCE PREMIUM CALCULATIONS \\ WITH ANTICIPATED UTILITY THEORY
}

\author{
BY \\ CUNCUN LUAN \\ Department of Mathematics \\ Nanjing University, Nanjing, China
}

\begin{abstract}
This paper examines an insurance or risk premium calculation method called the mean-value-distortion pricing principle in the general framework of anticipated utility theory. Then the relationship between comonotonicity and independence is explored. Two types of risk aversion and optimal reinsurance contracts are also discussed in the context of the pricing principle.
\end{abstract}

\section{KEYWORDS}

Mean-value-distortion pricing principle, anticipated utility theory, comonotonicity, risk aversion, and optimal reinsurance.

\section{INTRODUCTION}

The calculation of insurance or risk premiums has been an essential and active topic in actuarial literature, which has attracted the attention of actuaries such as Bühlmann (1970), Goovaerts et al. (1984) and Hürlimann (1997, 1998). Recently, modern theory of risk and economic choice under uncertainty has played an important role in studying insurance premium calculations (Wang et al., 1997, Wang and Young, 1998, Young, 1998). Hürlimann (1998) makes a brief, yet comprehensive summary about the development of insurance premium calculations. He emphasizes desirable and reasonable properties that insurance premiums should satisfy. In fact, most modern pricing principles, other than the distortion pricing principle, are presented in an expected utility framework, while Wang et al. (1997) applies Yaari' dual theory. However, both expected utility theory and Yaari' dual theory are special cases of anticipated utility theory (Puppe, 1991).

In this paper, the mean-value-distortion pricing principle is presented under anticipated utility theory as an approach to insurance premium calculations. This kind of premium calculation can be found in Denuit et al. (1999), which 
refers to Chateauneuf et al. (1997). An outline of the paper is as follows. In section 2, main properties of the mean-value-distortion pricing principle are investigated. It is shown that these properties are consistent with those of the mean value principle. Section 3 shows the relationship between independence and comonotonicity. Here risk aversion and optimal reinsurance are also discussed.

\section{Properties of The MEAN-VALUe-distortion Pricing PRINCIPLE}

\subsection{The Mean-Value-Distortion Pricing Principle}

Quiggin (1982) first discussed anticipated utility theory. Subsequently, Segal (1989) proposed an axiomatization of this theory, where the ordinal independence axiom substituted the independence axiom of expected utility theory. Analogous to Segal (1989), define "risk" as a non-negative random variable $X \in \Omega$ with distribution function $F_{X}(x)$ and survival function $S_{X}(x)$, where $x \geq 0$ and $\Omega=\{X: X \geq 0,0 \leq E X \leq \infty\}$. The insurance premium calculation is a nonnegative real function $\pi: \Omega \rightarrow R$. The premium of risk $X$ is denoted by $\pi(X)$.

Risks are restricted to bounded random variables and $\Pi=[0, M]$ is the domain of risks. Further, let $\preccurlyeq$ be a binary preference relation.

Axiom 1 (Weak Order): The relation $\preccurlyeq$ is weak order.

Axiom 2 (Continuity): For every risk $X$, the sets $\left\{F_{Y}(x): X \preccurlyeq Y\right\}$ and $\left\{F_{Y}(x)\right.$ : $Y \preccurlyeq X\}$ are closed in the topology of weak convergence.

Axiom 3 (Monotonicity): For all risks $X$ and $Y$, if $F_{X}(x) \geq F_{Y}(x), x \geq 0$, then $X \prec Y$.

Axiom 4 (Ordinal Independence): For all risks $X, X^{\prime}, Y$ and $Y^{\prime}$, if $F_{X}(x)=$ $F_{X^{\prime}}(x), F_{Y^{\prime}}(x)=F_{Y^{\prime}}(x)$ on $[0, c)$ (respectively on $[c, M]$ ) and $F_{X}(x)=F_{Y}(x)$, $F_{X^{\prime}}(x)=F_{Y^{\prime}}(x)$ on $[c, M]$ (respectively $[0, c)$ ), then $X \preccurlyeq Y \Leftrightarrow X^{\prime} \preccurlyeq Y^{\prime}$.

Preference relation $\preccurlyeq$ satisfies axioms $1,2,3$ and 4 if and only if there exists a continuous measure $\omega$ on $\Pi \times[0,1]$ with $\omega(A)>0$ for every non-empty open set $A \in \Pi \times[0,1]$ such that

$$
X \preccurlyeq Y \Leftrightarrow \omega\left(e_{X}\right) \leq \omega\left(e_{Y}\right)
$$

where epigraph $e_{X}$ is the closure of set $\left\{(x, p) \in \Pi \times[0,1]: p \geq F_{X}(x)\right\}$. The generalized utility function is defined by

$$
\bar{v}(x, p)=\omega([0, x] \times[1-p, 1]) \text { for all }(x, p) \in \Pi \times[0,1] .
$$

If the corresponding relative utility index $\frac{\bar{v}(x, \delta p)}{\bar{v}(x, p)}$ is independent of $x$ for all $\delta \epsilon$ $[0,1]$, then the preference relation $\preccurlyeq$ can be expressed by a real-valued functional:

$$
V(X)=-\int_{\Pi} v(t) d g\left(S_{X}(t)\right)
$$


where $v(x)$ and $g(x)$ are non-decreasing functions. Accordingly, the mean-valuedistortion pricing principle $\pi$ satisfies the following equation:

$$
v(\pi(X))=-\int_{\Pi} v(t) d g\left(S_{X}(t)\right)
$$

If $\lim _{d \rightarrow \infty} \pi(\min (X, d))=\pi(X)$, then the extension of equation (1) to $\Omega$ is given by

$$
v(\pi(X))=-\int_{0}^{\infty} v(t) d g\left(S_{X}(t)\right) .
$$

Integrating equation (2) by parts and assuming $\lim _{t \rightarrow \infty} v(t) g\left(S_{X}(t)\right)=0$ gives

$$
v(\pi(X))=\int_{0}^{\infty} g\left(S_{X}(t)\right) d v(t) .
$$

Obviously, if $g(x)=x$, equation (2) results in the mean value principle and if $v(x)=x$, equation (2') results in the distortion pricing principle. Since

$$
v(\pi(X))=-\int_{0}^{\infty} v(t) d g\left(S_{\pi(X)}(t)\right)=v(\pi(\pi(X))) .
$$

Hence equation (2) displays the certainty equivalent principle in which $\pi(X)$ is the sure payment leading to indifference. In the next part, the properties of mean-value-distortion pricing principle are developed.

\subsection{Properties}

Suppose that $v(x)$ is an increasing convex function, i.e., $v^{\prime}(x)>0, v^{\prime \prime}(x) \geq 0$, and $g(x)$ is an increasing concave and distortion function on $[0,1]$ such that $g(0)=0, g(1)=1$ and $g(x) \geq x$.

Theorem 2.1 (Non-Negative Loading): $\pi(X) \geq E X$ for all $X \in \Omega$.

Proof: Since $g(x) \geq x$ and $v(x)$ is convex,

$$
v(\pi(X))=\int_{0}^{\infty} g\left(S_{X}(t)\right) d v(t) \geq \int_{0}^{\infty} S_{X}(t) d v(t)=E(v(X)) \geq v(E X) .
$$

Thus, $\pi(X) \geq E X$.

Theorem 2.2 (Non-Excessive Loading): $\pi(X) \leq \sup (X)$ for all $X \in \Omega$. This result is obvious.

Theorem 2.3 (Scale Invariant): $\pi(k X)=k \pi(X)$ for all $k>0$ if and only if $v(x)=$ $a+b x^{\theta}$, where $a \in R, b>0$ and $\theta>0$.

Theorem 2.4 (Translation Invariant): $\pi(X+c)=\pi(X)+c$ for all $c \in R$ if and only if $v(x)=x$ or $v(x)=e^{r x}$ where $r>0$.

Before proving the prior two theorems, several lemmas from Goovaerts et al. (1984) are generalized. 
Lemma 2.1: Suppose $v(x)$ and $\tilde{v}(x)$ are continuous and increasing functions. For bounded risks, the sufficient and necessary condition such that $v(x)$ and $\tilde{v}(x)$ have the same solutions with respect to equation (1) is $\tilde{v}(x)=\alpha+\beta v(x)$, for all $x \in \Pi$ where $\alpha, \beta \in R$.

Proof: Assume $\tilde{\pi}(X)$ is a solution of equation (1) corresponding to $\tilde{v}(x)$. If for all $x \in \Pi, \tilde{v}(x)=\alpha+\beta v(x)$, and $\alpha, \beta \in R$, then

$$
\tilde{v}(\tilde{\pi}(X))=\alpha+\beta v(\pi(X)) \text { and } \tilde{v}(\tilde{\pi}(X))=\alpha+\beta v(\tilde{\pi}(X)) .
$$

So $v(x)$ and $\tilde{v}(x)$ have the same solutions. Conversely, let $X$ be a two-point random variable, i.e., $X=M$ with probability $q, X=0$ with probability $1-q$, where $0<q<1$. According to equation (2),

$$
\tilde{v}(\pi(X))=g(q) \tilde{v}(M)+(1-g(q)) \tilde{v}(0) \text { and } v(\pi(X))=g(q) v(M)+(1-g(q)) v(0)
$$

Since $1-g(q) \neq 0$, comparing the two equations above gives

$$
\frac{\tilde{v}(\pi(X))-\tilde{v}(M)}{v(\pi(X))-v(M)}=\frac{\tilde{v}(M)-\tilde{v}(0)}{v(M)-v(0)}
$$

This implies $\tilde{v}(x)=\alpha+\beta v(x)$ for $x \in[0, M]$, where $\alpha=\tilde{v}(M)-\frac{\tilde{v}(M)-\tilde{v}(0)}{v(M)-v(0)} v(M)$ and $\beta=\frac{\tilde{v}(M)-\tilde{v}(0)}{v(M)-v(0)}$.

Lemma 2.2: If $\lim _{d \rightarrow \infty} \pi(\min (X, d))=\pi(X)$ for all $d \geq 0$, lemma 2.1 also holds for risk $X \in \Omega$.

Proof of theorem 2.3: The "if" part is easy to verify. It suffices to prove the "only if" part. Since $\pi(k X)=k \pi(X)$ for all $k>0$,

$$
v(k \pi(X))=v(\pi(k X))=-\int_{0}^{\infty} v(k t) d g\left(S_{X}(t)\right) .
$$

Let $\tilde{v}(x)=v(k x)$, it follows that

$$
\tilde{v}(\pi(X))=v(k \pi(X))=-\int_{0}^{\infty} v(k t) d g\left(S_{X}(t)\right)=-\int_{0}^{\infty} \tilde{v}(t) d g\left(S_{X}(t)\right) .
$$

According to lemma 2.2, $\tilde{v}(x)=\alpha(k)+\beta(k) v(x)$ where $\alpha(k), \beta(k) \in R$ are dependent on $k$. Let $x=0, \tilde{v}(0)=\alpha(k)+\beta(k) v(0)$ and

$$
v(k x)-v(0)=\beta(k)[v(x)-v(0)] .
$$

Differentiating the above equation with respect to variable $k$,

$$
x v^{\prime}(k x)=\beta^{\prime}(k) v(x) \text { and } x^{2} v^{\prime \prime}(k x)=\beta^{\prime \prime}(k) v(x) .
$$

Finally, if $k=1$, then $\frac{v^{\prime \prime}(x)}{v^{\prime}(x)}=\frac{\beta^{\prime \prime}(1)}{\beta^{\prime}(1)} x$. This implies that $v(x)$ can be represented as $v(x)=a+b x^{\theta}$, where $a \in R, b>0$ and $\theta>0$. 
Proof of theorem 2.4: The "if" part is obvious by calculation. Conversely, assume $\pi(X+c)=\pi(X)+c$ for all $c \in R$. If $\tilde{v}(x)=v(x+c)$, then

$$
\tilde{v}(\pi(X))=v(\pi(X)+c)=v(\pi(X+c))=-\int_{0}^{\infty} v(t+c) d g\left(S_{X}(t)\right)=-\int_{0}^{\infty} \tilde{v}(t) d g\left(S_{X}(t)\right) .
$$

According to lemma 2.2, $\tilde{v}(x)=\alpha(c)+\beta(c) v(x)$ where $\alpha(c), \beta(c) \in R$ are dependent on $c$. Let $x=0, \tilde{v}(0)=\alpha(c)+\beta(c) v(0)$ and

$$
v(x+c)-v(0)=\beta(c)[v(x)-v(0)] .
$$

Differentiating the above equation with respect to variable $x$,

$$
v^{\prime}(x+c)=\beta(c) v^{\prime}(x) \text { and } v^{\prime \prime}(x+c)=\beta(c) v^{\prime \prime}(x) .
$$
Finally if $x=0$, then $\frac{v^{\prime \prime}(c)}{v^{\prime}(c)}=\frac{v^{\prime \prime}(0)}{v^{\prime}(0)}$ and $v^{\prime \prime}(0)=0$. It implies $v(x)=x$ otherwise,
$v(x)=e^{r x}$ where $r>0$.

Theorem 2.5 (Independent Additive): If risks $X$ and $Y$ are independent, $\pi(X+Y)$ $=\pi(X)+\pi(Y)$ if and only if $v(x)=x$ or $v(x)=e^{r x}$ where $r>0$ and $g(x)=x$.

Proof: The "if" part has been proved by Goovaerts et al. (1984). To prove the "only if" part, first note that the independent additive property implies that $\pi(X)$ satisfies translation invariance. Hence $v(x)=x$ or $v(x)=e^{r x}$ where $r>0$. If $v(x)=x$, then $\pi(X)=\int_{0}^{\infty} g\left(S_{X}(t)\right) d t$. Let risk $X \sim B(1, q)$, risk $Y \sim B(1, p)$ where $0 \leq p, q \leq 1$ and let risks $X$ and $Y$ be independent. Thus, $\pi(X)=g(q), \pi(Y)=g(p)$, $\pi(X+Y)=g(p+q-p q)+g(p q)$ and

$$
g(p+q-p q)+g(p q)=g(q)+g(p) .
$$

Differentiating equation (4) by argument $p$ and then $q$,

$$
g^{\prime \prime}(p+q-p q)(1-p)(1-q)-g^{\prime}(p+q-p q)+g^{\prime \prime}(p q) p q+g^{\prime}(p q)=0 .
$$

If $q=0$, then $g^{\prime \prime}(p)(1-p)-g^{\prime}(p)+g^{\prime}(0)=0$ and $g^{\prime}(0)=g^{\prime}(1)$.

If $q=1$, then $g^{\prime \prime}(p) p-g^{\prime}(1)+g^{\prime}(p)=0$.

Comparing (5) and (6), $g^{\prime \prime}(p)=0$ for all $p \in[0,1]$ which implies $g(x)=x$. Similarly, $g(x)=x$ if $v(x)=e^{r x}$ where $r>0$.

Theorem 2.6 (Comonotonic Additive): If risks $X$ and $Y$ are comonotonic, $\pi(X+Y)=\pi(X)+\pi(Y)$ if and only if $v(x)=x$.

Proof: The comonotonic additive property implies $\pi(X)$ preserves the scale and translation invariant properties. Therefore, it follows that $v(x)=x$ by theorem 2.3 and theorem 2.4 . 
Lemma 2.3 (Wang, 1998): For two comonotonic risks $X$ and $Y, \operatorname{Cov}(X, Y) \geq 0$. Let $E_{g}(X)=\int_{0}^{\infty} t d\left[1-g\left(S_{X}(t)\right)\right]$ and $\operatorname{Cov}_{g}(X, Y)=E_{g}(X Y)-E_{g}(X) E_{g}(Y)$.

Lemma 2.4: For two comonotonic risks $X$ and $Y, \operatorname{Cov}_{g}(X, Y) \geq 0$. The proof of this lemma is omitted since it roughly resembles that of lemma 2.3 .

Theorem 2.7 (Sub-Additive): For all risks $X$ and $Y, \pi(X+Y) \leq \pi(X)+\pi(Y)$ if and only if $v(x)=x$.

Proof: The "if" part has been proved by Hürlimann (1998). To prove the "only if" part, first note that the sub-additive property implies $\pi(X+c) \leq \pi(X)+\pi(c)$ for all $X$ and $c$. In addition, $\pi(c)=c$ by equation (2) and $\pi(X)=\pi(X+c-c) \leq$ $\pi(X+c)-\pi(c)$. Hence $\pi(X)$ is translation invariant. By theorem 2.4, $v(x)=x$ or $v(x)=e^{r x}$ where $r>0$. If $v(x)=e^{r x}$, then $\pi(X)=\frac{1}{r} \log \left[E_{g}\left(e^{r X}\right)\right]$ where $r>0$. Assuming risks $X$ and $Y$ are comonotonic, so are $e^{r X}$ and $e^{r Y}$. According to lemma 2.4 and theorem 2.6,

$$
E_{g}\left(e^{r X} e^{r X}\right)>E_{g}\left(e^{r X}\right) E_{g}\left(e^{r Y}\right) .
$$

That is, $\pi(X+Y)>\pi(X)+\pi(Y)$ for comonotonic risks.

Theorem 2.8 (Stop-Loss Order Preserving): If $X \prec_{s l} Y$, then $\pi(X) \leq \pi(Y)$.

The proof of this theorem refers to the third part of Hürlimann (1998). However, two points should be noted. One is that if $u=S_{X}(t)$ and $t=F_{X}^{-1}(1-u)$, equation (2) can be rewritten by

$v(\pi(X))=-\int_{0}^{\infty} v(t) d g\left(S_{X}(t)\right)=-\int_{0}^{1} v\left(F_{X}^{-1}(1-u)\right) d g(u)=\int_{0}^{1} v\left(F_{X}^{-1}(u)\right) d \gamma(u)$,

where $\gamma(u)=1-g(1-u)$. The second point is since $v(x)$ is an increasing convex function, then

$$
E(v(X)) \leq E(v(Y)) .
$$

Equation (3.5) from Hürlimann (1998), equations (7) and (8) from above all combine to show that the mean-value-distortion pricing principle regarding $\pi(X)$ preserves stop-loss order.

It is shown that essential properties of the mean-value-distortion pricing principle are consistent with corresponding properties of the mean value principle. These properties are more closely related to $v(x)$ than $g(x)$ because under anticipated utility theory, the effect of loss severity and loss probability is multiplicatively separable and

$$
v(\pi(X))=-\int_{0}^{\infty} v(t) d g\left(S_{X}(t)\right)=\int_{0}^{\infty} v(t) d\left[1-g\left(S_{X}(t)\right)\right] .
$$

The right-hand side of equation (9) can be viewed as the expected value of $v(X)$ with respect to $1-g\left(S_{X}(x)\right)$ instead of $F_{X}(x)$. Obviously $P_{g}=\left\{1-g\left(S_{X}(x)\right)\right\}$ is a 
probability space denoted as the distort-probability space. Here $\pi(X)$ may be regarded as the mean value premium of risk $X$ on $P_{g}$. In this light, the properties of the mean-value-distortion pricing principle should be different little from those of the mean value principle. It is believed that the distort-probability space $P_{g}$ is non-additive for independent risks, unless $g(x)=x$, but additive for comonotonic risks. Therefore, different additive properties among risks should be defined in different probability spaces when describing practical insurance operations.

\section{SOME RELATED CONCLUSIONS AND COMMENTS}

\subsection{The Relationship Between Independence and Comonotonicity}

In expected utility theory, independence is an important concept. In Yaari' dual theory, comonotonicity is stressed because of theoretical work and practical meanings. According to theorems 2.5 and 2.6, the mean-value-distortion pricing principle of $\pi(X)$ satisfies the independent additive and comonotonic additive properties if and only if $v(x)=x$ and $g(x)=x$. The following theorem (theorem 3.1) presents an alternative interpretation of the aforementioned result. Here the description of comonotonicity in Denneberg (1994) is applied. For further discussion regarding comonotonicity, one should refer to Schmeidler (1986) and Yaari (1987).

Lemma 3.1 (Denneberg, 1994): Risks $X$ and $Y$ are said to be comonotonic if there exist a risk $Z$ and increasing real-valued functions $f_{1}(x), f_{2}(x)$ such that

$$
X=f_{1}(Z) \text { and } Y=f_{2}(Z)
$$

Theorem 3.1: Risks $X$ and $Y$ are both independent and comonotonic if and only if one of them is a degenerate random variable.

Proof: Without loss of generality, let risk $X$ be a degenerate random variable. Obviously,

$$
F_{X, Y}(x, y)=F_{X}(x) F_{Y}(y)=\min \left\{F_{X}(x), F_{Y}(y)\right\} .
$$

Thus risks $X$ and $Y$ are independent and comonotonic. Conversely, assume risks $X$ and $Y$ are not degenerate random variables. By lemma 3.1, there exists a non-degenerate random variable $Z$ and increasing real-valued functions $f_{1}(x)$ and $f_{2}(x)$ such that $X=f_{1}(Z)$ and $Y=f_{2}(Z)$. Hence, $F_{X}(x)=F_{Z}\left(f_{1}^{-1}(x)\right)$ and $F_{Y}(y)=F_{Z}\left(f_{2}^{-1}(y)\right)$. Since risks $X$ and $Y$ are independent and comonotonic,

$$
\begin{aligned}
& F_{X}(x) F_{Y}(y)=F_{Z}\left(f_{1}^{-1}(x)\right) F_{Z}\left(f_{2}^{-1}(y)\right)=\min \left\{F_{Z}\left(f_{1}^{-1}(x)\right), F_{Z}\left(f_{2}^{-1}(y)\right)\right\} \\
& =F_{Z}\left(\min \left\{f_{1}^{-1}(x), f_{2}^{-1}(y)\right\}\right) .
\end{aligned}
$$


Let $t_{1}=f_{1}^{-1}(x)$ and $t_{2}=f_{2}^{-1}(y)$, it follows that

$$
F_{Z}\left(t_{1}\right) F_{Z}\left(t_{2}\right)=F_{Z}\left(\min \left\{t_{1}, t_{2}\right\}\right) .
$$

Finally assume $t_{1} \geq t_{2}$ and $t_{2} \rightarrow a$, where $\alpha$ is a lower bound of risk $Z, 0 \leq a \leq \infty$. It follows that

$$
F_{Z}\left(t_{1}\right)=1, t_{1} \geq \alpha \text { and } F_{Z}\left(t_{1}\right)=0, t_{1}<\alpha .
$$

This implies risk $Z$ is a degenerate random variable, which contradicts the assumption.

The above theorem illustrates that if non-degenerate risks are comonotonic, they must not be independent and vise versa. The next theorem provides a sufficient condition for determining whether risks are comonotonic or independent. Further, if risks are comonotonic, their sum may be easily obtained.

Theorem 3.2: If risks $X$ and $Y$ are comonotonic, their sum may be simplified as the addition of real-value functions, i.e.,

$$
X+Y=f_{1}(Z)+f_{2}(Z)=\left(f_{1}+f_{2}\right)(Z) .
$$

Proof: Let risks $X$ and $\eta$ have identical distributions, i.e. $F_{X}(x)=F_{\eta}(x)$ for all $x$. Thus,

$$
F_{X, \eta}(x, y)=\min \left\{F_{X}(x), F_{\eta}(y)\right\}=F_{X}(\min \{x, y\}) .
$$

If $x \leq y$ and $F_{X, \eta}(x, y)=F_{X}(x)$, then $\eta$ is constant and independent of risk $X$. It follows that

$P(X+f(\eta) \leq z)=\int_{x+f(x) \leq z} d F_{X, Y}(x, y)=\int_{x+f(x) \leq z} d F_{X}(x)=F_{X}\left[(1+f)^{-1} z\right]$.

Analogously, if $x>y$, equation (12) also exists. Thus, if $\eta=X$, then $f=f_{2} \circ f_{1}^{-1}$.

To examine a collective risk model, let

$$
X(t)=\sum_{i=1}^{N(t)} X_{i} \text { and } X(0)=0,
$$

where $\left\{X_{i}\right\}^{\infty} \in \Omega$ are independent claim sizes. $N(t)$ is the number of claims in the interval $[0, t]$ with $N(0)=0$ and $t>0$ independent of $\left\{X_{i}\right\}_{1}^{\infty}$. Risks $X_{1}, X_{2}$, ... may generate comonotonic risks $F_{X_{1}}^{-1}(\xi), F_{X_{2}}^{-1}(\xi), \ldots$, which have the same marginal distribution functions as risks $X_{1}, X_{2}, \ldots$, where $\xi \sim U(0,1)$. Risks $X_{i}$ and $F_{X_{i}}^{-1}(\xi)$ belong to the same individual risk group and $\sum_{i=1}^{N(t)} X_{i} \prec_{s l} \sum_{i=1}^{N(t)} F_{X_{i}}^{-1}(\xi)$, then $\pi\left(\sum_{i=1}^{N(t)} X_{i}\right) \leq \pi\left(\sum_{i=1}^{N(t)} F_{X_{i}}^{-1}(\xi)\right)$. Hence, the portfolio consisting of comonotonic risks determines an upper bound of insurance premiums that may be viewed as a market price. Insurance companies should not price risks above this market price. 


\subsection{Risk Aversion}

Wang (1996), Wang and Young (1998) distinguish between two types of risk aversion. One type is based on an individual's attitude towards wealth under expected utility theory while the other is based on varying probabilities under dual theory. The authors believe that insurance entities reflect different levels of risk aversion based on their sizes. In fact, there is one type of risk aversion under both expected utility theory and dual theory. The difference is presented in their actual expressions. Puppe (1991, p. 67) argues this point:

"Two concepts of risk aversion will be considered here. The first concept defines an individual to be risk averse if the sure gain $E(F)$ of the expectation of a distribution $F$ is always preferred to the distribution itself. An alternative definition of risk aversion. suggested by Rothschild and Stiglitz (1970), requires a risk averse individual to prefer a distribution $F$ to any mean preserving spread of $F$."

The two definitions of risk aversion are equivalent only under expected utility theory. In regards to insurance pricing theory, an insurer's pricing principle reflects its attitude towards risks. Insurers who are risk averse expect their pricing principles to preserve stop-loss order, which is consistent with the second definition of risk aversion particularly under non-expected utility theory. To avoid any confusion, the second definition is preferred. It is also known that risk aversion is equivalent to the convexity of $v(x)$ under expected utility theory, to the concavity of $g(x)$ under dual theory, and to both of them under anticipated utility theory.

Dual theory parallels expected utility theory from the standpoint of utilizing probabilities versus wealth. Even so, risk aversion based on expected utility theory and risk aversion based on dual theory cannot be compared. This result can be seen by the characterization theorem of comparative risk aversion discussed by Puppe (1991, p. 71). Hence considering only the size of an insurer is insufficient in determining which pricing principle an insurer should utilize. The following theorems clarify the aforementioned risk aversion comparisons.

Lemma 3.2: Let $\bar{V}$ and $\bar{V}^{*}$ be rank-dependent utility functionals with corresponding generalized utility functions $\bar{v}$ and $\bar{v}^{*}$, respectively. Assume $\bar{v}_{12}$ and $\tilde{v}_{12}^{*}$ exist everywhere and are differentiable with respect to both arguments. Then, $\bar{V}$ is more risk averse than $\bar{V}^{*}$ if and only if for all $(x, p) \in \Pi \times[0,1]$ the following two relations hold.

$$
\frac{\bar{v}_{121}(x, p)}{\bar{v}_{12}(x, p)} \leq \frac{\bar{v}_{121}^{*}(x, p)}{\bar{v}_{12}^{*}(x, p)} \text { and } \frac{\bar{v}_{122}(x, p)}{\bar{v}_{12}(x, p)} \geq \frac{\bar{v}_{122}^{*}(x, p)}{\bar{v}_{12}^{*}(x, p)} .
$$

(Note the prior assumptions regarding $v(x)$ and $g(x)$ are implied in the following theorems.)

Theorem 3.3: An insurer is more risk averse under expected utility theory than under dual theory if and only if $\frac{\nu^{\prime \prime}(x)}{v^{\prime}(x)} \leq 0$ and $\frac{g^{\prime \prime}(p)}{g^{\prime}(p)} \leq 0$ for all $x \in \Pi$ and $0 \leq p \leq 1$. 
Theorem 3.4: An insurer is more risk averse under expected utility theory and dual theory than under anticipated utility theory.

Within the same theoretical system, it is true that the degree of risk aversion is closely related to the size of an insurer, i.e., its wealth. This is the concept of decreasing risk aversion. In general, decreasing risk aversion implies "an individual with utility function $u(x)$ is more risk averse than another one with utility function $u(w+x), w>0$, under the standard of maximizing expected utility functions". The Arrow-Pratt measure of risk aversion, $r(x)$, is a decreasing function of $x$ applicable to expected utility theory. However, this measure does not make any sense in dual theory because here the Arrow-Pratt measure is zero. According to lemma 3.2, under anticipated utility theory "the characterization of decreasing risk averse is exactly the same as in the expected utility model."

The above discussion of risk aversion stems from an insurer's point of view. However, from an insured's perspective, results will be perfectly opposite. Arguably, insurance is the outcome of high-speed development of an economy. The result is the existence of a luxury commodity, insurance, which allows an individual to exchange uncertain outcomes for a certain one after having certain wealth accumulation. In addition, the more wealth an individual has, the more care they are likely to place in the insurance market.

\subsection{Optimal Reinsurance}

From an insurance company's perspective, the optimization criterion of a reinsurance contract is to minimize the insurance premium of retained risks. A reinsurance contract $I^{*}(X) \in I$ is said to be an optimal reinsurance contract with respect to the pricing principle $\pi$ if $\pi\left[X-I^{*}(X)\right]<\pi[X-I(X)]$ for all $I(X) \in I$, where $I=\left\{I(x): I(0)=0,0 \leq I^{\prime} \leq 1\right\}$ is a set of reinsurance contracts. The most useful two subsets of $I$ are $I_{\pi, P}, I_{\mu}$, where $I_{\pi, P},=\left\{I(x): I(0)=0,0 \leq I^{\prime} \leq 1, \pi[I(X)]\right.$ $=P\}, I_{\mu}=\left\{I(x): I(0)=0,0 \leq I^{\prime} \leq 1, E[I(X)]=\mu\right\}$, and $P, \mu$ are fixed. Goovaerts et al. (1990) gives an informative exposition regarding optimal reinsurance in the case of $I_{\mu}$. Wang (1998) and Young (1999) study this problem with respect to the distortion pricing principle.

Lemma 3.2 (Goovaerts et al., 1990): For any optimization criterion preserving stop-loss order, the optimal reinsurance contract over set $I_{\mu}$ is of the form $I^{*}(X)=(X-d)_{+}$and is called the stop-loss contract.

Theorem 3.5: According to the mean-value-distortion pricing principle, the stop-loss contract is the optimal reinsurance contract for $I_{\mu}$ and $I_{g, \mu}$, where $I_{g, \mu}=\left\{I: I(0)=0,0 \leq I^{\prime} \leq 1, E_{g}[I(X)]=\mu\right\}$.

Proof: Applying theorem 2.8 and lemma 3.2, it is easy to prove the result for $I_{\mu}$. Further, according to equation $(9), v(\pi(X))=E_{g}[v(X)]$ and since $v^{\prime \prime} \geq 0$,

$$
v(t)-v(z) \geq v^{\prime}(z)(t-z) \text { for all } t, z \in R .
$$


Therefore, $v[x-I(x)]-v\left[x-I^{*}(x)\right] \geq v^{\prime}\left[x-I^{*}(x)\right]\left[I^{*}(x)-I(x)\right]$. If less $I^{*}(x)-I(x)>0$, then $x-I^{*}(x)=d$ and

$$
v[x-I(x)]-v\left[x-I^{*}(x)\right] \geq v^{\prime}(d)\left[I^{*}(x)-I(x)\right] .
$$

Otherwise, $x-I^{*}(x) \leq d, v^{\prime}\left[x-I^{*}(x)\right] \leq v^{\prime}(d)$ and inequality (13) also exists. Substituting $X$ for $x$ in inequality (13) and integrating both sides with respect to $1-g\left(S_{X}(x)\right)$ yields

$$
v[\pi(X-I(X))]-v\left[\pi\left(X-I^{*}(X)\right)\right] \geq v^{\prime}(d) E_{g}\left[I^{*}(X)-I(X)\right]=0
$$

This implies $I^{*}(X)=(X-d)_{+}$is the optimal reinsurance contract.

Corollary 3.1: If $\pi(X)=\pi_{g}(X)=\int_{0}^{\infty} g\left(S_{X}(t)\right) d t$, then $I_{\pi, P}=I_{g, P}$. In this case the stoploss contract is the optimal reinsurance contract for $I_{\mu}$ and $I_{\pi, P}$ according to the distortion pricing principle.

For the mean-value-distortion pricing principle, the problem of an extreme value with respect to $\pi[X-I(X)]$ is identical to $v[\pi(X-I(X))]$. In this case a larger set $I_{g, P}^{\tilde{v}}=\left\{I(x): I(0)=0,0 \leq I^{\prime} \leq 1, E_{g}[\tilde{v}(I(X))]=P\right\}$ is considered, where $\tilde{v}(x)$ make the integral exist. Obviously $I_{\mu}, I_{\pi, P}$ and $I_{g, \mu}$ are all subsets of $I_{g, P}^{\bar{v}}$. Moreover, since $v[\pi(x-I(x))]$ is Gâteaux differentiable with respect to $I(x)$, the method of resolving a constrained extreme value of a functional to find an optimal reinsurance contract is applied.

Theorem 3.6: In the set $I_{g, P}^{\tilde{v}}$, an optimal reinsurance contract $I^{*}(x)$ is determined by the equation $v^{\prime}[x-I(x)]=\lambda \bar{v}^{\prime}[I(x)]$, where $\lambda$ satisfies the constraint $E_{g}\left[\tilde{v}\left(I^{*}(X)\right)\right]$ $=P$.

Proof: Let $\varphi(I)=E_{g}[v(X-I(X))]$ and $\Phi(I)=E_{g}[\tilde{v}(I(X))]$. The aim is to minimize $\varphi(I)$ under the constraint $\Phi(I)=P$. Let $f(I, \lambda)=\varphi(I)-\lambda \Phi(I)$. For all real $t$ and functional $h(x)$, optimal reinsurance $I^{*}(x)$ satisfies $\lim _{t \rightarrow 0} \frac{f(I+t h, \lambda)-f(I, \lambda)}{t}=0$.
That is,

$$
\lim _{t \rightarrow 0} \frac{\varphi(I+t h)-\varphi(I)-\lambda[\Phi(I+t h)-\Phi(I)]}{t}=0 .
$$

Substituting for $\varphi(I)$ and $\Phi(I)$ in equation (14), we have

$$
E_{g}\left\{\left[v^{\prime}(X-I(X))-\lambda \tilde{v}^{\prime}(I(X))\right] h(X)\right\}=0 \quad \text { for all functional } h(x) .
$$

Thus, $v^{\prime}[x-I(x)]=\lambda \tilde{v}^{\prime}[I(x)]$ for all $x \geq 0$, where $\lambda$ satisfies $E_{g}\left[\tilde{v}\left(I^{*}(X)\right)\right]=P$.

Corollary 3.2: The optimal reinsurance contract $I^{*}(x)$ for $I_{\pi, P}$ is determined by $v^{\prime}[x-I(x)]=\lambda v^{\prime}[I(x)]$, where $\lambda$ satisfies the constraint $\pi\left[I^{*}(X)\right]=P$.

Corollary 3.3: If $v(x)=x^{2}$, the optimal reinsurance contract for $I_{\pi, P}$ is a quota share contract. 


\section{CONCLUSION}

This paper discusses the insurance or risk premium calculation known as the mean-value-distortion pricing principle in the general framework of anticipated utility theory. Essential properties such as non-negative loading, non-excessive loading, scale and translation invariant, stop-loss order preservation, and sub-additivity are preserved in the analysis of the pricing principle. It is also shown that for non-degenerate risks, independence and comonotonicity do not exist simultaneously. Risk aversion is not comparable under expected utility theory and Yaari's dual theory. This fact suggests consideration of insurance problems in a larger theoretical frame. Finally, optimal reinsurance contracts are derived by different computational methods.

\section{ACKNOWLEDGEMENTS}

The author gratefully acknowledges anonymous referees for their helpful comments and suggestions. The author is also grateful to Professor Jean Lemaire and Mr. Martin Halek from the Wharton School of the University of Pennsylvania for their work regarding the English language.

\section{REFERENCES}

Bühlmann, H. (1970) Mathematical Methods in Risk Theory. Springer-Verlag.

Chateauneuf, A., Cohen, M. and Meilisson I. (1997) Comonotonicity, rank-dependent utilities and a search problem. In: Benes, V. and Stepan J. (Ed.). Distributions with given marginals and moment problems. Kluwer Academic Publishers, Amsterdam.

Denneberg, D. (1994) Non-Additive Measure and Integral. Kluwer Academic Publishers, Boston.

Denurt, M., Dhaene, J. and Van Wouve, M. (1999) The Economics of Insurance: a review and some recent developments. Bulletin of the Swiss Association of Actuaries, 137-175.

GoovaerTS, M.J., De Vylder, F. and HaEzendonck J. (1984) Insurance Premiums: Theory and Applications. North-Holland, Amsterdam.

Goovaerts, M.J., KaAs, R., Van HeerwaArden, A.E. and Bavwelinck, X.T. (1990) Effective Actuarial Methods. North-Holland, Amsterdam.

HürlimanN, W. (1997) On quasi-mean value principles. Blätter der Deutschen Gesellschaft für Versicherungsmathematik XXIII, 1-16.

HüRLIMANN, W. (1998) On stop-loss order and the distortion pricing principles. ASTIN Bulletin 28(2), 119-134.

PupPE, C. (1991) Distorted Probabilities and Choice under Risk. Springer-Verlag.

Quiggin, J. (1982) A theory of anticipated utility. Journal of Economic Behavior and Organization 3, 323-343.

RothSChild, M. and STiglitz J.E. (1970) Increasing risk: I. A Definition. Journal of Economic Theory 2, 225-243.

SEGAL, U. (1989) Anticipated utility theory: a measure representation approach. Annals of Operations Research 19,359-373.

SCHMEIDLER, D. (1986) Integral representation without additivity. Proceedings of American Mathematical Society 97, 255-261.

WANG, S. (1996) Premium calculation by transforming the layer premium density. ASTIN Bulletin 26(2), 71-92. 
WAng, S., Young, V.R. and PANJer H.H. (1997) Axiomatic characterization of insurance prices. Insurance: Mathematics and Economics 21,173-183.

WANG, S. (1998) An actuarial index of the right-tail risk. North American Actuarial Journal 2(2), $89-101$.

WANG, S. and YouNG V.R. (1998) Ordering of risks: utility theory verse Yaari's dual theory of risk. Insurance: Mathematics and Economics 22, 145-161.

YAARI, M.E. (1987) The dual theory of choice under risk. Econometrica 55, 95-115.

YOUNG,V.R. (1998) Families of updated rules for non-additive measures: Applications in pricing risks. Insurance: Mathematics and Economics 23, 1-14.

Young, V.R. (1999) Optimal insurance under Wang's premium principle. Insurance: Mathematics and Economics 25, 109-122.

\author{
CUNCUN LUAN \\ Department of Mathematics \\ Nanjing University \\ Nanjing 210093 \\ P.R. China \\ Email:Luancc@263.net
}

